\title{
Lidil
}

Revue de linguistique et de didactique des langues

$59 \mid 2019$

Prononcer les langues : variations, émotions, médiations

\section{Amir Biglari et Marc Bonhomme (dir.), La présupposition entre théorisation et mise en discours} Paris, Classiques Garnier, collection « Rencontres », n 350, 2018, 582 p. Laure Gardelle

\section{OpenEdition Journals}

Édition électronique

URL : http://journals.openedition.org/lidil/6181

DOI : $10.4000 /$ lidil.6181

ISSN : $1960-6052$

Éditeur

UGA Éditions/Université Grenoble Alpes

Édition imprimée

ISBN : 978-2-37747-090-7

ISSN : $1146-6480$

Référence électronique

Laure Gardelle, « Amir Biglari et Marc Bonhomme (dir.), La présupposition entre théorisation et mise en discours », Lidil [En ligne], 59 | 2019, mis en ligne le 01 mai 2019, consulté le 25 septembre 2020. URL http://journals.openedition.org/lidil/6181; DOl : https://doi.org/10.4000/lidil.6181

Ce document a été généré automatiquement le 25 septembre 2020.

(C) Lidil 


\title{
Amir Biglari et Marc Bonhomme (dir.), La présupposition entre théorisation et mise en discours
}

Paris, Classiques Garnier, collection « Rencontres », $\mathrm{n}^{\circ} 350,2018,582$ p.

\author{
Laure Gardelle
}

\section{RÉFÉRENCE}

Amir Biglari et Marc Bonhomme (dir.), La présupposition entre théorisation et mise en discours, Paris, Classiques Garnier, collection « Rencontres », n 350, 2018, 582 p.

1 Cet ouvrage collectif, qui compte vingt-deux contributions en plus d'une solide présentation générale, s'intéresse à la définition et aux manifestations de la "présupposition", concept qui, malgré de nombreuses utilisations du terme en linguistique et plusieurs ouvrages de synthèse, n'est pas encore stabilisé aujourd'hui. Selon l'approche, selon l'époque, le «centre de fixation" n'est pas le même: le présupposé est condition de vérité en logique, élément de contenu implicite, complémentaire du posé, en linguistique, tandis qu'en pragmatique, il ne se limite pas au langagier, mais s'étend aux interactions. L'extrême diversité des marqueurs ne facilite pas la tâche. Tous s'accordent à dire que le présupposé présente deux caractéristiques: il est pré-requis pour la compréhension des énoncés (il s'agit d'implicite à valeur littérale), et dépend du posé, avec lequel il entretient une relation dissymétrique (il «fournit le cadre informatif d'arrière-plan sur lequel le posé se déploie »). Mais au-delà, les contours sont flous : par exemple, faut-il inclure, comme le font certains, l'hyperonymie? Quelles différences avec le «sous-entendu», les « implicatures » conversationnelles ? Le présupposé existe-t-il au-delà de l'échelle de la phrase?

2 L'ouvrage propose cinq axes d'étude du concept de présupposition. La première partie (6 chapitres) propose des approches théoriques; les autres constituent des cas 
pratiques. La deuxième partie (3 contributions) étudie le fonctionnement de la présupposition dans des structures syntaxiques (structures concessives, constructions pseudo-clivées) et le discours sur la grammaire des remarqueurs du XvII ${ }^{\mathrm{e}}$ siècle. La partie 3 (4 études) approfondit les exploitations littéraires de la présupposition, la partie 4 (6 contributions) les exploitations dans le discours politico-médiatique ; enfin, la dernière partie ( 3 chapitres) explore l'applicabilité du concept en sémiotique de l'image. Ce dernier volet est encore à un stade embryonnaire dans ce champ disciplinaire ; les trois contributions montrent son opérabilité.

Une recension ne peut faire justice à la richesse des analyses développées; voici à titre d'exemples quelques idées qui ont particulièrement retenu l'attention du présent auteur. D'un point de vue théorique, Louis de Saussure et Jacques Moeschler soulignent tous deux des différences fondamentales entre présupposé (explicature, sans guère de marge interprétative) et implicite (implicatures, ce que le locuteur « veut dire " par un biais indirect, cf. Sperber \& Wilson). Saussure distingue deux formes de présupposition: linguistique (déclenchée localement) et discursive (déclenchée globalement); Jacques Moeschler établit une distinction entre présupposition sémantique et pragmatique, et entre implicatures conventionnelles et conversationnelles. Ces différences peuvent être établies en partie linguistiquement par des tests, dont celui bien connu de la négation, d'applicabilité variable cependant, ou la «loi d'enchaînement» de Ducrot. Sandrine Deloor et Jean-Claude Anscombre ajoutent que dans l'approche énonciative qui est la leur, décrire le sens d'un énoncé, c'est décrire « la façon dont cet énoncé représente la réalité »; le présupposé doit donc être un élément de contenu de l'énoncé, par rapport auquel le posé est asserté (voir de même Marion Carel). Ils critiquent en cela l'approche référentialiste, majoritaire actuellement, pour laquelle décrire le sens d'un énoncé, c'est décrire la réalité à laquelle il réfère, et donc pour laquelle la présupposition serait « une relation entre un énoncé et une croyance d'arrière-plan ». Pour Catherine Kerbrat-Orrechoni, posé, présupposé et implicature (ou « sous-entendu ») sont en fait tous des unités de contenu de l'énoncé, et c'est l'ensemble de ces trois composantes qui en constitue le sens global. Alain Rabatel montre par ailleurs que certains présupposés sont moins «nécessaires » que d'autres, ou encore que les présupposés lexicaux sont plus «volatiles » que les présupposés syntaxiques; si présupposés et sous-entendus diffèrent, il faut distinguer une forme de "gradualité ». Notamment, «bien des présupposés donnent naissance à des sous-entendus, en fonction du principe de pertinence ».

Une autre idée forte de l'ouvrage est le rôle potentiellement argumentatif et manipulatoire des présupposés. Ainsi, le problème de notre société égalitariste implique de faire admettre que notre société est égalitaire, et que c'est un problème (Saussure). C'est la perspective discursive, notamment le marquage d'éléments présupposés comme étant syntaxiquement secondaires (Jacques Jayez \& Robert Reinecke), qui crée cet effet. Ce rôle argumentatif du présupposé est finement décodé grâce à différentes études pratiques, avec micro-analyses en contexte, notamment via l'analyse des concessives dans la presse (Corinne Rossari), des pseudo-clivées (Mervi Helkkula), du discours publicitaire (Karine Berthelot-Guiet), des Contemplations de Victor Hugo, qui font beaucoup appel au vous (Amir Biglari), ou encore l'étude des parenthèses (si l'on peut dire) lors du débat télévisé entre François Hollande et Nicolas Sarkozy (présidentielle 2012), régulièrement détournées de leur fonction de rappel consensuel (Ruggero Druetta). 
5 En somme, cet ouvrage très accessible, d'une grande clarté et assorti de nombreux exemples d'application, de discussions, de ressources bibliographiques dans divers cadres théoriques, sera très profitable à tout lecteur (chercheur, étudiant ou personne intéressée par le langage) désireux de faire le point sur la part de sens des énoncés qui ne relève pas du posé, de l'explicite. La diversité des contributions, ainsi que l'équilibre entre apports théoriques et analyses pratiques, et les tentatives d'extension à la sémiotique, en font une contribution forte aux recherches actuelles.

\section{AUTEURS}

\section{LAURE GARDELLE}

LIDILEM, Université Grenoble Alpes 